\title{
Análise experimental da resistência dos nós na osteossíntese tipo banda de tensão
}

Experimental analysis of knot resistance in tension band osteosynthesis

Ricardo Bonato', Carlos Alberto Morato', Fabio Lucas Rodrigues'

\begin{abstract}
Resumo
Introdução: Realizou-se estudo experimental com objetivo de avaliar a resistência de nós de fios metálicos confeccionados com tensionador de fios A0ASIF (Grupo 1) comparados àqueles realizados com torção manual (Grupo 2), na síntese do tipo tirante de tensão. Método: Foram utilizados 28 corposde-prova de nylon 6,6 com $32 \mathrm{~mm}$ de diâmetro e $50 \mathrm{~cm}$ de comprimento com uma secção transversal incompleta de $27 \mathrm{~mm}$ no centro, formando uma zona de tensão na face íntegra do tarugo. Os fios foram aplicados aos tarugos e dois tipos de nós foram utilizados (Grupos 1 e 2). 0 conjunto foi montado em máquina de ensaios mecânicos de tração/compressão até fadiga da síntese, com mensuração da tensão. Resultados: 0 resultado estatístico da tensão no momento de fadiga, segundo média, desvio padrão, mediana, tensão mínima e máxima, demonstrou que o grupo 1 possui um nó $50 \%$ menos resistente à distração que o grupo 2. Conclusão: 0 s resultados mostraram que o tensionador de fios A0-ASIF produz um nó cerca de $50 \%$ menos resistente à distração quando comparado ao nó de torção manual.
\end{abstract}

Palavras-chave: Engenharia biomédica; fixação interna de fraturas; imobilização; estresse mecânico; patela.

\section{Abstract}

Introduction: An experimental study aiming at evaluating the resistance of metallic threads knots made using a thread tensionator A0-ASIF (Group 1) was accomplished in order to compare them to those made with manual torsion (Group 2) in the tension strut type synthesis. Method: Twenty-eight proof bodies were used. They consisted of nylon 6.6 with $32 \mathrm{~mm}$ of diameter and $50 \mathrm{~cm}$ of length with an incomplete transversal section of $27 \mathrm{~mm}$ in the center, forming a tension zone in the pin's complete face. The threads were applied to the pins and two-knot types were used (Groups 1 and 2). The proof body and cerclage were set up in a traction/compression mechanical assays machine till synthesis fatigue, measuring the tension. Results: Tension statistical results obtained in the fatigue moment, using the average, deviation standard, medium, minimum and maximum tension, demonstrated that Group 1 has a knot 50\% less resistant to the distraction than Group 2. Conclusion: The results showed that the thread tensionator A0-ASIF produces a knot almost $50 \%$ less resistant to the distraction when compared to the manual torsion knot.

Keywords: Biomedical engineering; fracture fixation; immobilization; stress, mechanical; patella.

Recebido: 16/8/2009

Revisado: 16/12/2009

Aprovado: $28 / 1 / 2010$

Trabalho realizado no Hospital Estadual Mario Covas de Santo André, Santo André (SP), Brasil.

Setor de Trauma Ortopédico do Hospital Estadual Mario Covas de Santo André, Santo André (SP), Brasil.

Endereço para correspondência: Fabio Lucas Rodrigues - Hospital Estadual Mário Covas -Rua Doutor Henrique Calderazzo, 321 - Valparaíso -

CEP 09190-615 - Santo André (SP), Brasil - e-mail: fabio.lucas.rodrigues@hotmail.com 


\section{Introdução}

A industrialização trouxe benefícios à humanidade e, com ela, as crescentes lesões por traumas ou avulsões. $\mathrm{O}$ aumento dos graves traumatismos da mão decorrentes de acidentes com máquinas, explosivos e veículos, em que existe alta concentração de energia cinética, é progressivo e cada vez mais grave.

O objetivo da osteossíntese é promover estabilidade que permita a mobilização precoce, além de se obter bom posicionamento dos fragmentos fraturados ${ }^{1}$. A escolha do tipo de redução ou do material de síntese a ser empregado depende do padrão da fratura, da qualidade óssea, da idade e do grau de atividade do paciente ${ }^{1,2}$.

Dessa maneira, é imperativo buscar recursos que posam prover um restabelecimento do membro lesionado e que o torne funcional o mais breve possível, iniciando rapidamente o processo de reparação tecidual associado à reabilitação. Isso implica buscar tipos de osteossínteses que consigam resistir às forças deformantes que atuam em cada um desses segmentos e que dê preferência àqueles que oferecem resistências próximas aos valores dos ossos íntegros.

Neste sentido, a técnica de osteossíntese com banda de tensão é um dos possíveis métodos empregados para o tratamento de fraturas por avulsão. As osteossínteses podem ser realizadas por fios de aço, placas metálicas em aço, Vitallium e Titânio, por parafusos interfragmentários e, desde a década de 1990, por placas bioabsorvíveis. Esta técnica foi descrita difundida por Muller et al. ${ }^{3}$, consiste numa amarração com fio metálico colocado na face de tensão da fratura a qual tem sido recomendada ao longo das décadas, sendo replicada por diversos cirurgiões, tais quais, Benjamin ${ }^{4}$, Ostrum $^{5}$ e Fan 6 .

Esse princípio baseia-se no fato de que cargas excêntricas aplicadas em uma barra geram tensão na superfície convexa e compressão na superfície côncava, que permite imediata mobilização do segmento afetado e retorno precoce do paciente às suas atividades cotidianas.

Seu uso mais frequente é nas fraturas articulares por avulsão, como na patela, no olecrano e no maléolo medial. A técnica cirúrgica iniciase com estabilização dos fragmentos fraturários com fios de Kirschner paralelos, os quais transfixam os fragmentos proximal e distal da fratura. Fixa-se um fio maleável metálico aos dois fragmentos fraturários, fazendo que adquira a forma de um "8", unidos em suas extremidades por um nó, que pode ser realizado por duas técnicas diferentes: torção manual ou torção com tensionador de fios AO-ASIF (também conhecido como "foguetinho").

Essa técnica, então, é relatada por vários cirurgiões ${ }^{3,6}$ em estudos experimentais, buscando descrever a força ideal no enrolamento do nó destinado a manter a tensão do fio metálico. Entretanto, pouco se investigou sobre o tipo de nó destinado a manter essa força. Em face dessa contínua necessidade de desenvolvimento de técnicas menos invasivas e mais funcionais, este trabalho teve como objetivo comparar a resistência máxima suportada pelo nó realizado com torção manual com aquela suportada pelo nó realizado com tensionador de fios AO-ASIF ("foguetinho”).

\section{Método}

O experimento consiste na montagem de um modelo de fratura óssea fixado com uma síntese tipo banda de tensão (cerclagem). O osso fraturado é simulado por um tarugo de nylon e a síntese é realizada com fio de aço. Esse conjunto denomina-se corpo-de-prova e é submetido a uma força de angulação até fadiga da síntese.

São 28 tarugos de nylon $6,6 \mathrm{com} 32 \mathrm{~mm}$ de diâmetro e $50 \mathrm{~cm}$ de comprimento. Em todos, há uma seção transversal de $27 \mathrm{~mm}$ distando $25 \mathrm{~cm}$ da extremidade. A seguir são feitos furos de $2 \mathrm{~mm}$ de diâmetro interessando ambos os córtices e perpendiculares ao plano longitudinal passante diametralmente pela porção íntegra do tarugo. Tais furos localizam-se $1 \mathrm{~cm}$ abaixo do córtice que está íntegro (na face de tensão) e a $5 \mathrm{~cm}$ do corte transversal incompleto, conforme demonstrado na Figura 1.

Nos furos dos tarugos são introduzidos os fios de aço de cerclagem. O experimento é dividido em dois grupos, segundo a espessura do fio de aço: nas medidas 1,2 e 1,5 mm. Cada grupo é subdividido em dois novos grupos, variando o tipo de nó: o primeiro feito com o tensionador de fios AO-ASIF e o segundo com a torção manual das duas pontas do fio de aço.

O nó realizado com o tensionador inicia-se com a manufatura de uma argola em uma extremidade do fio. Passa-se a outra extremidade através da argola, prendendo-a no tensionador que possui um mecanismo rotatório para tensionar o fio. Depois de realizada a tensão, dobrase o fio a 180 graus sobre seu eixo (Figura 2).

Já o nó manual realiza-se com o envolvimento das duas pontas livres utilizando porta agulha. São confeccionadas cinco voltas entre as extremidades. Finaliza-se com tensionamento torcendo o nó com alicate (Figura 3).

Os corpos-de-prova são montados num dispositivo de cutelos com roletes, onde os inferiores estão posicionados a $19 \mathrm{~cm}$ de distância e o superior alinhado no ponto médio do tarugo. Imprimiu-se uma velocidade de descida de $10 \mathrm{~mm} / \mathrm{min}$ que impõe o direcionamento da carga perpendicularmente na face oposta da cerclagem (Figura 4). O

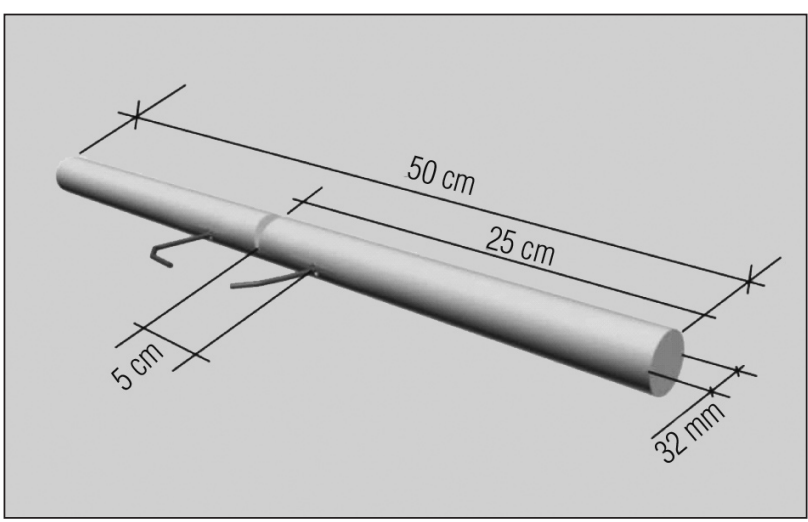

Figura 1 - Desenho esquemático do corpo de prova, com dimensões em centímetros. 
mecanismo de mensuração da força necessária para fadiga da síntese se dá por meio de uma máquina de ensaios mecânicos de tração/compressão marca MTS de acionamento por fuso, utilizando célula de carga de 2000 kgf monitorada por um sistema HP Vectra VE DX-2 66 com software Q-test v1.0 m.

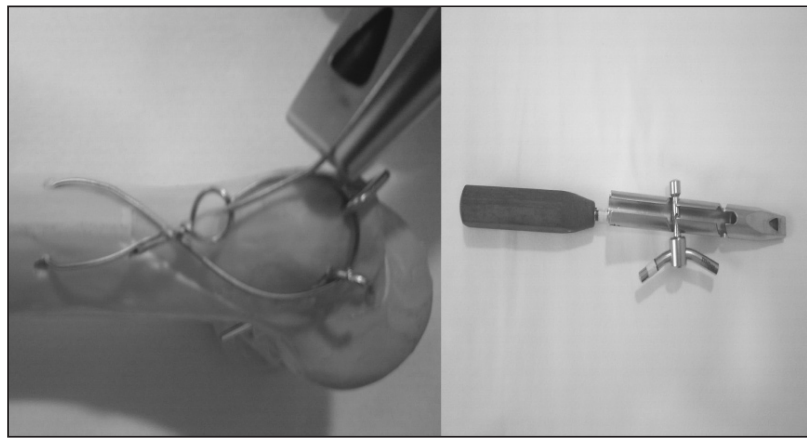

Figura 2 - Modelo de cerclagem com tensionador de fios A0-ASIF.

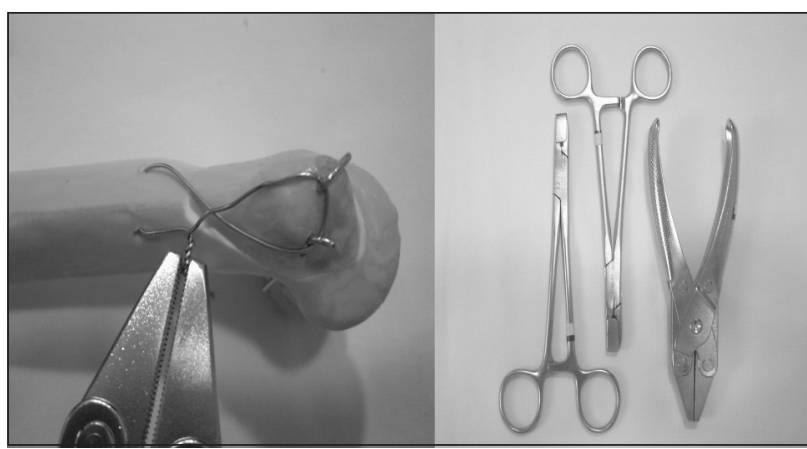

Figura 3 - Modelo de cerclagem com torção manual e material utilizado.
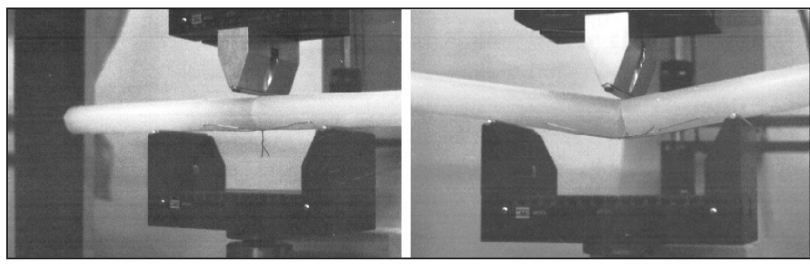

Figura 4 - Sequência de teste aplicada no corpo de prova.

\section{Resultados}

Os resultados estão representados nas Tabelas I e II, onde figuram a média e o desvio padrão segundo as variáveis acima descritas. Nas provas relacionadas às duas espessuras de fios foram encontradas diferenças estatisticamente significantes em relação às metodologias de amarração e à carga máxima. No caso do fio de $1,2 \mathrm{~mm}$, a média obtida pela torção manual foi muito maior que a obtida pelo tensionador de fios AO-ASIF, e a razão entre elas é 1,93 (52,85:27,41). Também para o fio $1,5 \mathrm{~mm}$ a torção manual conseguiu melhor média em relação ao tensionador de fios AO-ASIF, com uma razão de 1,52 $(77,90: 51,40)$

\section{Discussão}

A osteossíntese ideal deve prover estabilidade para manter o foco de fratura sem nenhuma imobilização externa. A força de compressão conseguida pela técnica de tirante colocado na face de tensão promove tal objetivo ao permitir a imediata mobilização articular e favorecer a consolidação com melhor função.

Há uma ideia geral de que o tratamento das fraturas de metacárpicos e falanges deva ser conservador sempre que possível. Todavia, com frequência variável, o tratamento cirúrgico se impõe geralmente pela impossibilidade de manutenção da redução por meios externos. Entretanto, não existe consenso sobre qual o melhor método de fixação de fraturas instáveis de metacárpicos e falanges, estando à escolha de um ou outro método na dependência da preferência pessoal de cada cirurgião, mais por uma questão de vivência do que de confirmação científica da superioridade do método escolhido.

Relatos de Rayhack et al. ${ }^{7}$ demonstraram a superioridade das fixações com bandas de tensão, comparadas aos fios de Kirschner cruzados. Já Vanik et al. ${ }^{8}$ realizaram estudos comparativos de várias técnicas de osteossíntese em metacárpicos humanos embalsamados, observando que as cerclagens são superiores às montagens somente com fios de Kirschner. Também Viegas et al. ${ }^{9}$ estudaram seis diferentes configurações de fixações com fios de Kirschner em fraturas transversal e oblí-

Tabela I - Resultado estatístico de ensaio realizado com fio de 1,2 mm de espessura - tensionador de fios A0-ASIF/manual

\begin{tabular}{lllcccc}
\hline & Média" & DP* & Mediana & Mínimo & Máximo & $N^{* *}$ \\
\hline Tensionador manual & $52,85^{*}$ & 6,40 & 53,80 & 44,40 & 59,00 & 7 \\
Tensionador A0-ASIF & $27,41^{\prime \prime}$ & 4,84 & 26,10 & 21,40 & 36,20 & 7 \\
\hline
\end{tabular}

* Desvio padrão; ** quantidade de amostras utilizadas.

Unidades de mediana mínimo máximo e $\mathrm{N} ; \mathrm{T}=8,50$ e $\mathrm{p}<0,001$.

Tabela II - Resultado estatístico de ensaio realizado com fio de 1,5 mm de espessura - tensionador de fios A0-ASIF/manual

\begin{tabular}{lcccccc}
\hline & Média " & DP* & Mediana & Mínimo & Máximo & $N^{* *}$ \\
\hline Tensionador Manual & $77,90^{*}$ & 7,18 & 78,80 & 65,50 & 86,40 & 7 \\
Tensionador A0-ASIF & $51,40^{\prime \prime}$ & 3,79 & 51,10 & 47,60 & 58,70 & 7 \\
\hline
\end{tabular}

* Desvio padrão; ${ }^{* *}$ quantidade de amostras utilizadas.

Unidades de mediana mínimo máximo e N; $\mathrm{T}=8,64$ e p $<0,001$ 
quas de falanges, demonstrando a melhor estabilidade nas fixações com quatro fios de Kirschner, cruzados dois a dois.

Dessa maneira, vários relatos são disponíveis na literatura sobre as osteossínteses, porém aqueles relacionados com análises experimentais da resistência dos nós na osteossíntese tipo de tensão são escassos. Além disso, outros autores estudaram diferentes tipos de osteossíntese, chegando a resultados nem sempre concordantes, embora houvesse clara superioridade da banda de absorção de tensão, porém a questão da tensão ideal não é relatada.

Neste estudo, envolvendo a comparação da resistência máxima suportada pelo nó realizado com torção manual com aquela suportada pelo nó realizado com tensionador de fios AO-ASIF ("foguetinho"), observou-se que as características dos nós podem perder-se em caso de sua soltura ou quando a tensão do tirante não prover compressão adequada. Por outro lado, a tensão não pode ser excessiva, sob pena de quebra da amarra por diminuição da elasticidade ${ }^{10}$ ou retardo na consolidação ${ }^{11,12}$. Esses fatores devem ser considerados na escolha da técnica de amarração.

A torção manual promoveu um nó mais resistente a deslocamentos secundários, mas essa é uma técnica menos suscetível à padroni- zação, tanto da tensão aplicada no fio maleável, que acaba variando de acordo com a força aplicada pelo cirurgião, como da técnica de enrolamento dos fios, podendo conter um número pequeno de voltas. Outra possível falha na técnica é aplicar menor tensão em um dos fios, a qual leva um fio torcer-se sobre o outro, o que torna o nó menos resistente.

$\mathrm{O}$ uso do tensionador de fios AO-ASIF facilita o tensionamento do fio de cerclagem; o nó obtido, entretanto, mostrou-se, neste estudo, cerca de 50\% menos resistente à distração, ou seja, com relevante possibilidade de falha na osteossíntese.

Assim, conclui-se que o nó confeccionado por torção manual foi mais resistente que o realizado com tensionador de fios AO-ASIF ("foguetinho").

Destaca-se que os diferentes tipos de osteossíntese oferecem estabilidades diferentes e apresentam características próprias. Sua escolha depende fundamentalmente do tipo de fratura, da habilidade do cirurgião, dos recursos e do tipo de material disponíveis, devendo sempre buscar aquela que melhor se adapta aos seus objetivos. Entretanto, na aplicação da tensão, a escolha do tipo de osteossíntese pareceu ser um fator que pode interferir no resultado final do procedimento cirúrgico.

\section{Referências}

1. lannottiJP, Ramsey ML, Williams GR Jr, Warner JJ. Nonprosthetic management of proximal humeral fractures. Instr Course Lect. 2004;53:403-16.

2. Broos PL, Semon A. From unstable internal fixation to biological osteosynthesis. A historical overview of operative fracture treatment. Acta Chir Belg. 2004;104(4):396-400.

3. Müller $M E$, Allgöver $M$, Schneider R, Willenegger H. A Patela e a tíbia, fraturas da patela. In: Manual de Osteossintese. Técnicas recomendadas pelo grupo A0-ASIF. São Paulo, Manole, 1993;13: 564-7.

4. Benjamin J, Bried J, Dohm M, McMurtry M. Biomechanical evaluation of various forms of fixation transverse patellar fractures. J Orthop Trauma. 1987;1(3):219-22.

5. Ostrum RF, Litsky AL. Tension band fixation of medial malleolus fractures. $J$ Orthop Trauma. 1992;6(4):464-8.

6. Fan $\mathrm{GF}, \mathrm{Wu} \mathrm{CC}$, Shin $\mathrm{CH}$. Olecranon fractures treated with tension band wiring techniques - comparisons among three different configurations. Changgeng Yi Xue Za Zhi. 1993;16(4):231-8.
7. Rayhack JM, Belsole RJ, Skelton WHJr. A strain recording model: analysis of transverse osteotomy fixation in small bones. J Hand Surg Am. 1984;9(3):383-7

8. Vanik RK, Weber RC, Matloub HS, Sanger JR, Gingrass RP. The comparative strengths of internal fixation techniques. J Hand Surg Am. 1984;9(2): 216-21.

9. Viegas SF, Ferren EL, Self J, Tencer AF. Comparative mechanical properties of various Kirschner wire configurations intransverse and oblique phalangeal fractures. J Hand Surg Am. 1988;13(2):246-53.

10. McGreal G, Reidy D, Joy A, Mahalingam K, Cashman WF. The biomechanical evaluation of polyester as a tension band for the internal fixation of patellar fractures. J Med Eng Technol.1999;23(2):53-6.

11. Engel EE, Volpon JB, Shimano AC. Mechanical testing of the tension band wire fixation in the proximal femur. Arch Orthop Trauma Surg. 1997;116(5):266-70.

12. Terjesen $\mathrm{T}$, Svenningsen $\mathrm{S}$. The effects of function and fixation stiffness on experimental bone healing. Acta Orthop Scand. 1988;59(6):712-5. 